\title{
EVOLUÇÃO ELETRENCEFALOGRÁFICA EM MULHERES COM EPILEPSIA DE DIFÍCIL CONTROLE
}

\author{
ANA CLÁUDIA S. G.B.AZOUBEL * DIOSELY C. SILVEIRA *, CARLOS A.M. GUERREIRO*
}

RESUMO - Foram revistos 444 traçados eletrencefalográficos realizados durante o acompanhamento de 62 mulheres do Ambulatorio de Epilepsia Catamenial do Hospital das Clínicas da UNICAMP com diagnóstico de epilepsia de difícil controle, que compareceram a esse ambulatório regularmente por um período mínimo de cinco anos. Dos $350(78,84 \%)$ traçados anormais, 273 mostraram atividade epileptiforme e 77 anormalidades "não-epileptiformes". Segundo definiçōes adotadas em relação à frequência das crises, encontramos 18 pacientes (29\%) que apresentavam crises frequentes, $16(25,8 \%)$ com crises muito frequentes, $12(19,3 \%)$ com crises esporádicas e $16(25,8 \%)$ com crises que se tomaram controladas durante o período de acompanhamento. Em relaçăo aos achados de EEG, quatro pacientes $(6,5 \%)$ mostraram EEGs sempre normais, $30(48,4 \%)$ tiveram EEGs normais e anormais e $28(45,2 \%)$ apenas EEGs anormais. Entre aquelas cujos EEGs sempre foram normais, duas estavam com crises controladas, uma com crises esporádicas e uma com crises frequentes. Das 30 pacientes com EEGs normais e anormais, 10 estavam controladas, 5 tinham crises esporádicas, 9 tinham crises frequentes e 6 tinham crises muito frequentes. No grupo de pacientes com EEGs sempre anormais, 4 estavam controladas, 6 tinham crises esporádicas, 8 tinham crises frequentes e 10 tinham crises muito frequentes. Em relaçāo ao último EEG, ele foi normal em $7(43,7 \%)$ das 16 pacientes que estavam controladas, em $3(25 \%)$ das 12 pacientes com crises esporádicas, em $7(38,9 \%)$ das 18 pacientes com crises frequentes e em nenhuma das pacientes com crises muito frequentes. Os pacientes que persistem com EEGs normais parecem ter melhor evolução que aqueles que persistem com EEGs anormais. Pela análise deste estudo, pode-se sugerir um papel relativo do EEG quanto ao prognóstico a longo prazo no controle das crises epilépticas, pois as pacientes que persistiran com EEGs normais aparentemente tiveram evolução clínica mais favorável que aquelas que persistiram com os EEGs alterados.

PALAVRAS-CHAVE: EEG, epilepsia na mulher, epilepsia refratária.

\section{Electroencephalographic evolution in women with medically refractary epilepsy}

SUMMARY - We reviewed 444 EEGs of 62 women with medically refractary epilepsy, followed up for at least 5 years and that had 5 or more EEGs. According to our definitions we found 18 patients $(29 \%)$ with frequent seizures, $16(25.8 \%)$ with very frequent seizures, $16(25.8 \%)$ with controlled seizures and $12(19.3 \%)$. with occasional seizures. Four patients $(6.5 \%)$ always showed normal EEGs, $30(48.4 \%)$ had normal and abnormal EEGs and $28(45.2 \%)$ only abnormal EEGs. Among the patients who had only normal EEGs, two had all seizures controlled, one had occasional seizures and one had frequent seizures. Among the patients who had normal and abnormal EEGs, 10 had controlled seizures, 5 had occasional seizures, 9 had frequent seizures and 6 had very frequent seizures. In the group of patients with always abnormal EEGs, 4 had controlled seizures, 6 had occasional seizures, 8 had frequent seizures, and 10 had very frequent seizures. In relation to the last EEG, it was normal in $7(43.7 \%)$ of 16 patients with controlled seizures, in $3(25 \%)$ of 12 patients with occasional seizures and in 7 (38.9\%) of 18 patients with frequent seizures, and in none of the patients with very frequent seizures. The patients who had only normal EEGs seem to have a better outcome than those with abnormal EEGs. We observed that the last EEG was normal in $43.7 \%$ of the patients with controlled seizures. These data may suggest a relative importance of the EEG considering the long-term prognosis regarding seizure control.

KEY WORDS: EEG, epilepsy in women, refractary epilepsy.

*Setor de Epilepsia e Neurofisiologia Clínica do Departamento de Neurologia da Faculdade de Ciências Médicas (FCM) da Universidade Estadual de Campinas (UNICAMP). Aceite: 20-março-1995.

Dr. Carlos A.M. Guerreiro - Departamento de Neurologia, FCM/UNICAMP - Caixa Postal 6111 - 13081-970 Campinas SP - Brasil. 
O eletrencefalograma (EEG) é considerado o exame complementar mais sensível e útil na avaliação diagnóstica das epilepsias. Porém, não está bem definido o papel do EEG no seguimento de pacientes epilépticos ${ }^{9}$. As evidências clínicas demonstrando especificidade das descargas epileptiformes do EEG para epilepsias se iniciaram com o estudo de Zivin e Ajmone-Marsan (1968). Eles demonstraram que somente 2,2\% dos indivíduos não epilépticos apresentavam EEG com espículas e ondas agudas. Destes, $14,1 \%$ desenvolveram crises subsequentemente ${ }^{12}$. Este mesmo grupo de investigadores (Ajmone-Marsan e Zivin, 1970), analisando 1824 EEGs de 308 pacientes epilépticos, encontrou traçados sempre alterados em $30 \%$ dos pacientes, traçados normais e anormais em $51 \%$ e consistentemente normais em 17,5\%. Após um ano, 8\% continuavam com EEGs normais. Não houve relação entre os resultados do EEG e o tipo de crise epiléptica, exceção à crise parcial complexa de origem temporal, em que apenas $2 \%$ apresentavam registros exclusivamente negativos 1 . Apesar de controverso, alguns autores acreditam que o EEG tenha implicações prognósticas na decisão da retirada da medicação ${ }^{35}$.

Realizamos o presente estudo com o objetivo de avaliar a frequência de alterações eletrencefalográficas, o tipo e persistência destes achados em pacientes com epilepsias de difícil controle.

\section{CASUÍSTICA E MÉTODOS}

Avaliamos a evolução clínica c eletrencefalográfica de 62 pacientes do Ambulatório de Epilepsia Catamenial do Hospital das Clínicas da Universidade Estadual de Campinas (UNICAMP), originalmente em número de 80 pacientes $^{6}$. Como critério de inclusão a paciente devia referir pelo menos uma crise no mês anterior à consulta da inclusão no seguimento. $O$ tempo mínimo de acompanhamento ambulatorial foi de cinco anos. Todas as pacientes apresentavam epilepsia de difícil controle inicialmente e nenhuma apresentava retardo mental ou distúrbio comportamental relevante.

\section{Características da população do estudo}

A idade das 62 pacientes variou de 23 a 55 anos, com média de 37,23 anos.

Em relação às epilepsias, a idade de início das crises epilépticas variou de 0 a 30 anos, com média de 26,94 anos e o tempo de doença entre 10 e 51 anos, com média de 26,94 anos.

Trinta e sete $(59,7 \%)$ pacientes apresentavam epilepsias criptogênicas, 21 (33,9\%) epilepsias sintomáticas e $4(6,4 \%)$ epilepsias idiopáticas.

Seis $(9,67 \%)$ pacientes apresentavam crises generalizadas do tipo tônico-clônico (CGTC) e/ ou ausência e $56(90,32 \%)$ crises parciais com ou sem generalização secundária, predominando as crises parciais complexas (CPC) em $51(91,03 \%)$ destas 56 pacientes.

\section{Métodos}

Foram revistos, retrospectivamente, os resultados de todos os EEGs das pacientes, realizados no Serviço de Neurofisiologia Cínica do Hospital de Clínicas da UNICAMP, inclusive um estudo de quatro EEGs realizados em intervalos semanais durante um ciclo menstrual, à época da admissāo dessas pacientes no ambulatório. Os EEGs anormais foram considerados "epileptiformes" (AE) quando mostraram grafoelementos do tipo espículas, ondas agudas e complexos espícula-onda lenta e "não epileptiformes" (NAE), quando mostraram atividade lenta contínua ou intermitente. No referido serviço, rotineiramente, os traçados têm duração média de 30 minutos.

Os EEGs foram registrados em aparelhos de 8 e 16 canais, sob vigília ou sono espontâneo, utilizando eletrodos de superfície de acordo com o Sistema Internacional 10-20 de colocação de 
eletrodos, com registros mono e bipolares e complementados com técnicas de ativação por hiperventilação e fotoestimulação intermitente.

Tabela I: Frequência de crises na última consulta e número de EEGs realizados durante 5 anos de acompanhamento.

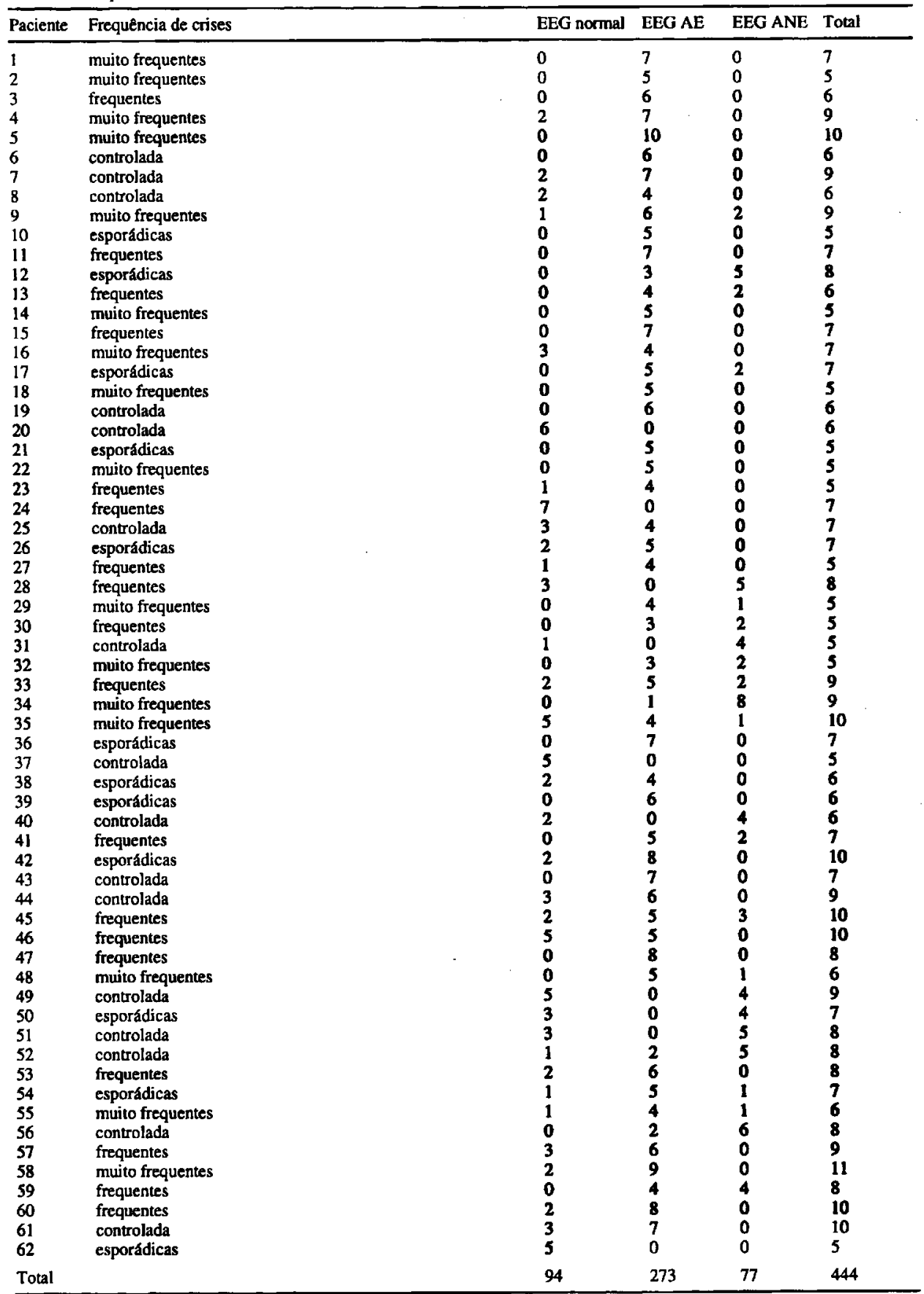


Quanto à frequência de crises epilépticas, baseados nos relatos das pacientes durante a última consulta e nos registros em calendário recente, foram consideradas: "controladas" as pacientes sem crises epilépticas há pelo menos um ano; com crises "esporádicas" as pacientes que apresentaram crises em intervalos entre 30 dias e um ano; com crises "frequentes" aquelas que apresentaram crises em intervalos entre 7 e 30 dias; e "muito frequentes" em intervalos menores que 7 dias.

\section{RESULTADOS}

Foram realizados 444 traçados, com média de 7,16 exames por paciente, variando de 5 a 11 exames. Destes, $94(21,2 \%)$ foram normais e $350(78,8 \%)$ anormais. Duzentos e setenta e três EEGs mostraram AE e 77 NAE.

Das 62 pacientes, $16(25,8 \%)$ estavam controladas, $12(19,3 \%)$ apresentavam crises esporádicas, $18(29 \%)$ apresentavam crises frequentes e $16(25,8 \%)$ crises muito frequentes (Tabela 1$).$

Evolução dos EEGs

O primeiro EEG foi anormal em $46(74,1 \%)$ das pacientes. Após a realização de quatro EEGs seriados, 49 (79\%) mostraram EEGs anormais. No período de acompanhamento ambulatorial por cinco anos, com pelo menos cinco EEGs realizados, 58 (93,5\%) mostraram EEGs anormais.

Em quatro pacientes $(6,5 \%)$ os EEGs sempre foram normais, em $30(48,4 \%)$ os EEGs mostraram-se ora normais e ora anormais e em 28 pacientes os EEGs foram sempre anormais .

\section{Evolução clínica em relaçāo aos EEGs}

Entre as quatro pacientes cujos EEGs foram sempre normais, duas estavam controladas, uma com crises esporádicas e uma com crises frequentes. Entre as 30 pacientes que mostraram EEGs normais e anormais, 10 estavam controladas, 5 tinham crises esporádicas, 9 tinham crises frequentes e 6 tinham crises muito frequentes. No grupo das 28 pacientes com EEGs sempre anormais, 4 estavam controladas, 6 com crises esporádicas, 8 com crises frequentes e 10 com crises muito frequentes (Tabela 2).

Resultado do último EEG em relaf̧ão à frequência de crises

Entre as 16 pacientes controladas, em $7(43,7 \%)$ o EEG foi normal e em $9(56,3 \%)$ foi anormal, com AE em 8 e NAE em 1 . No grupo das 12 pacientes com crises esporádicas, o EEG foi normal em $3(25 \%)$ e anormal em $9(56,3 \%)$, com AE em 8 e NAE em 1 . Das 18 pacientes com crises frequentes,

Tabela 2. Evolução e tipo dos EEGs; frequência de crises.

\begin{tabular}{lccccc}
\hline $\begin{array}{l}\text { Frequência de } \\
\text { crises }\end{array}$ & $\begin{array}{l}\text { Pacientes com } \\
\text { EEG normal }\end{array}$ & $\begin{array}{l}\text { Pacientes com EEG } \\
\text { normal/anormal }\end{array}$ & $\begin{array}{l}\text { Pacientes com EEG } \\
\text { anormal }\end{array}$ & Total \\
\hline Controlada & $20-37$ & $7-8-25-31-40-44-49-52-61$ & $6-19-43-56$ & $N^{\circ}$ & $\%$ \\
Esporádicas & 62 & $26-38-42-50-54$ & $10-12-17-21-36-39$ & 16 & 25,8 \\
Frequentes & 24 & $23-27-28-33-45-46-53-57-60$ & $3-11-13-15-30-41-47-59$ & 18 & 29 \\
Muito frequentes & 0 & $4-9-16-35-55-58$ & $1-2-5-14-18-22-29-32-34-48$ & 16 & 25,8 \\
Total & $4(6,5 \%)$ & $30(48,4 \%)$ & $28(45 \%)$ & 62 & 100 \\
\hline
\end{tabular}


Tabela 3. Último EEG e frequência de crises.

\begin{tabular}{|c|c|c|c|c|c|}
\hline \multirow{2}{*}{$\begin{array}{l}\text { Frequência de } \\
\text { crises }\end{array}$} & \multirow{2}{*}{$\begin{array}{l}\text { Pacientes com EEG } \\
\text { normal }\end{array}$} & \multirow{2}{*}{$\begin{array}{l}\text { Pacientes com atividade } \\
\text { epileptiforme }\end{array}$} & \multirow{2}{*}{$\begin{array}{l}\text { Pacientes com } \\
\text { atividade não } \\
\text { epileptiforme }\end{array}$} & \multicolumn{2}{|c|}{ Total } \\
\hline & & & & $\mathbf{N}^{\circ}$ & $\%$ \\
\hline Controlada & $7-20-25-31-37-51-52$ & $6-8-19-40-43-44-49-61$ & 56 & 16 & 25,8 \\
\hline Esporádicas & $42-50-62$ & $10-12-17-21-26-36-38-39$ & 54 & 12 & 19,4 \\
\hline Frequentes & $23-24-27-28-45-46-53$ & $3-11-13-30-41-47-57-60$ & $13-33-59$ & 18 & 29 \\
\hline Muito frequentes & 0 & $1-2-4-5-16-22-25-29-34-35-48-58$ & $9-14-32-55$ & 16 & 25,8 \\
\hline Total & $17(27,4 \%)$ & $36(58,0 \%)$ & $09(14,6 \%)$ & 62 & 100 \\
\hline
\end{tabular}

o EEG foi normal em $7(38,9 \%)$ e anormal em $11(61,1 \%)$, com AE em 8 e NAE em 3. O EEG mostrou-se anormal nas 16 pacientes com crises muito frequentes (100\%), 12 com AE e 4 com NAE (Tabela 3).

\section{COMENTÁRIOS}

É alta a correlação entre atividades potencialmente epileptogênicas ao EEG e a ocorrência de eventos ictais na história clínica do paciente?. O EEG interictal de rotina em pacientes epilépticos geralmente demonstra alterações sugestivas de epilepsia em $50 \%$ a $60 \%$, durante o primeiro exame. Quando repetidos, as chances de detectar anormalidades aumentam, podendo chegar a 92\% quando os traçados de sono são incluídos ${ }^{1,2,11}$. Neste estudo, foram revistos, retrospectivamente, todos os EEGs realizados durante os cinco anos de acompanhamento de 62 mulheres com crises epilepticas de difícil controle medicamentoso. Em 74\% das pacientes, o primeiro EEG foi anormal. Apos a realização de quatro EEGs no período de um ciclo menstrual, foram observadas anormalidades em 79\% destas pacientes. Outros EEGs foram realizados no período de cinco anos, quando $93,5 \%$ das pacientes mostraram anormalidades eletrencefalográficas. Estes achados estão de acordo com Binnie ${ }^{2}$ e Ajmone-Marsan e Zivin' ${ }^{1}$ que observaram que as chances de detectar anormalidades aumentam com a repetição de exames de EEG, podendo chegar a $92 \%$ quando se inclui EEG sob sono. Entretanto, 6,5\% das pacientes persistiram com EEGs sempre normais. Este achado também está de acordo com os estudos anteriores, que encontraram EEGs normais, mesmo após repetição do exame, em 5 a $8 \%$ dos pacientes ${ }^{12,211}$.

O encontro de EEGs normais nessas pacientes poderia decorrer da ausência de captação da descarga de um foco mais profundo pelo eletrodo de superfície ${ }^{10}$, ou da curta duração do traçado do EEG de rotina em relaçāo às 24 horas do dia para captar descargas que podem ocorrer ao acaso ou infrequentemente.

Parece haver, proporcionalmente, maior concentração de pacientes epilépticas controladas e com crises esporádicas entre aquelas cujos EEGs foram sempre normais, em relação às pacientes que evoluiram com EEGs normais e anormais durante os 5 anos de acompanhamento clínicoeletrencefalográfico e às pacientes cujos exames foram sempre anormais. Este fato poderia sugerir um melhor prognóstico para este grupo de pacientes.

Quando se considera o resultado do último EEG, a maior porcentagem de EEGs normais entre as pacientes que estão controladas $(43,7 \%)$ sugere um melhor prognóstico para esse grupo de pacientes.

Numa revisão realizada por Dean e Penry os autores comentam que a maioria dos investigadores concorda que o EEG, em associação com outros fatores importantes, tais como idade de início, 
severidade das crises e etiologia das epilepsias, auxiliariam na determinação do prognóstico dos pacientes epilépticos ${ }^{5}$.

Com este estudo pudemos constatar que, mesmo entre epilépticos de difícil controle, persiste um pequeno número que mantém os EEGs normais, apesar de não haver dúvidas quanto à condição epiléptica desse grupo. Nesses casos, justifica-se uma investigação neurofisiológica com técnicas especiais de registro, através de procedimentos semi-invasivos como o uso de eletrodos miniesfenoidais ou esfenoidais e até invasivos, como os eletrodos de profundidade ${ }^{8}$. A telemetria, por sua vez, permite a monitorização necessária para que se estabeleça um diagnóstico e se faça um planejamento terapêutico, quando indicado ${ }^{7,11}$.

Os pacientes que persistem com EEGs normais parecem ter evolução mais favorável do que aqueles que persistem com os EEGs alterados, mais frequentes entre pacientes com crises intratáveis clinicamente ou só tratáveis do ponto de vista cirúrgico.

Pela análise deste estudo, pode-se sugerir que o acompanhamento dos pacientes com EEGs, pelo menos anuais, poderia contribuir do ponto de vista prognóstico. Observamos ainda um elevado indice de anormalidades eletrencefalograficas ( $74 \%$ ) no primeiro EEG, provavelmente por se tratarem de pacientes com epilepsia de difícil controle.

\section{REFERÊNCIAS}

1. Ajmone-Marsan C, Zivin L. Factors related to the occurrence of typical paroxysmal abnormalities in the EEG records of epileptic patients. Epilepsia 1970, 11:361-381.

2. Binnie CD. Electrencephalography. In Laidlaw J, Richens A, Oxley J. (eds). A textbook of epilepsy: London: Churchill Livingstone, 1988, p 236-306.

3. Cardoso TMO, Guerreiro CAM. Quando iniciar e interromper as drogas antiepilépticas. In Guerreiro CAM Guerreiro MM (eds). Epilepsia. São Paulo: Lemos-Editorial, 1993, p 143-150.

4. Daly DD. Epilepsy and syncope. In Daly DD, Pedley TA (eds). Current practice of clinical electroencephalophy. New York: Raven Press, 1990, p 269-334.

5. Dean JC, Penry JK. Discontinuation of antiepileptic drugs. In Levy R, Melbrum B, Penry FK, Dreifuss FE (eds). Antiepileptic drugs. Ed 3. New York: Raven Press, 1989, p 133-142.

6. Guerreiro CAM, Ramos MC. Premenstrual seizure increase: influence of age, duration of disease, seizure frequency, previous complaint of perimenstrual accentuation, EEG and CT findings. Arq Neuropsiquiatr 1991, 49:27-32.

7. Ives JR. Ambulatory EEG monitoring: current state of the art of event type recorders and their future applications. Ambulat Monit, 1989, 2:183-189.

8. Ives JR, Schomer DL, Blume HW. Presurgical evaluations using cassete EEG. In Ebersole JS (ed). Ambulatory EEG monitoring. New York: Raven Press, 1989, p 195-215.

9. Pedley TA. The EEG in epilepsy adults: Clinical EEG (course 244). American Academy of Neurology, May, 1992, p 120.

10. Sharbrough FW. Scalp-recorded ictal patterns in focal epilepsy. J Clin Neurophysiol. 1993, 10:262-267.

11. Shorvon SD. The routine EEG. In Dam M, Gram L (eds). Compreensive epileptology. New York: Raven Press, 1990, p 321-338.

12. Zivin L, Ajmone-Marsan C. Incidence and prognostic significance of non-epileptic subjects. Brain 1968, 91:751-778. 